\title{
多维腕力传感器时域动态建模*
}

\author{
徐 科军 \\ (合肥工业大学电气系, 合肥 230009)
}

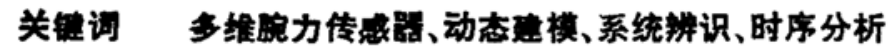

多维腕力传感器是智能机器人中最重要的传感器之一, 检测三维空间的力和力矩信息, 使 机器人准确无误地完成机械装配、精密磨削、轮廓跟踪等工作. 腕力传感器应用于机器人力控 制系统中, 这要求它响应速度快、动态品质好. 但目前, 该传感器的动态性能难以满足机器人 实时控制的要求, 国内外在此方面的研究相当薄弱. 通过分析与实验, 本文认为在腕力传感器 动态特性研究中存在三个关键问题:

1. 如何建立准确的动态数学模型;

2. 如何克服动态标定中设备的制约和方法的局限;

3. 如何改善动态性能指标.

其中, 第一个问题最为基本. 动态数学模型定量描述传感器输人、输出关系, 是研究其工 作机理的重要手段. 依据数学模型, 可正确评定传感器的动态性能; 可进行数字仿真, 预测传 感器对不同形式输人量的响应, 解决实验研究中的困难和局限; 可设计动态补偿数字滤波器, 消除动态误差, 提高动态性能指标. 而目前仅把腕力传感器简单地等效为二阶系统, 与实际情 况相差甚远. 为此, 本文依据实验数据, 分别采用系统辦识方法和时序分析方法, 从时域角度 研究腕力传感器的动态响应, 建立准确的动态数学模型, 为其性能指标计算、数字仿真和动态 补偿提供可靠的理论依据.

\section{1 时城动态标定}

传感器动态建模包括理论方法和实验方法两大类. 实验法分时间域和频率域, 均要基于 动态标定实验数据. 动态标定方法有多种, 本文选用脉冲响应法. 此方法较易实现, 效果较 好. 将 SAFMS 型系列六维腕力传感器安装在动态标定测试台上, 用摆锤对传感器施加冲击 力. 锤头中装有压电式传感器, 经电荷放大器, 将激励传感器的冲击力转换成电信号, 送人 MC 6600 计算机数据采集系统. 腕力传感器在冲击力作用下, 输出六路信号: 三路代表力分 量, 三路代表力矩分量, 其中, 与冲击力同向的力分量输出信号幅度最大. 数据采集系统以 5 $\mathrm{kHz}$ 或 $10 \mathrm{kHz}$ 的采样频率, 记录下这七路信号.

\section{2 系统辩识法建摸}

系统辨识就是根据传感器的输人、输出数据, 加以数据处理和数学计算, 估计出传感器的 动态模型 ${ }^{[1]}$. 辩识方法有多种, 本文采用最小二乘法. 实践证明, 此方法效果很好.

1993-03-09 收稿, 1993-06-29 收监改稿。

“国家自然科学基金和国家“863”网点实验室基金资助项目. 
以差分方程作为传感器的数学模型

$$
A\left(z^{-1}\right) y(k)=B\left(z^{-1}\right) u(k),
$$

式中 $A\left(z^{-1}\right)=1+a_{1} z^{-1}+a_{2} z^{-2}+\cdots+a_{1} z^{-1}$,

$$
B\left(z^{-1}\right)=b_{0}+b_{1} z^{-1}+b_{2} z^{-2}+\cdots+b_{1} z^{-1} .
$$

考虑到模型误差的存在, 传感器的测量方程为

$$
A\left(z^{-1}\right) y(k)=B\left(z^{-1}\right) u(k)+v(k),
$$

式中 $v(k)$ 为残差.

要对实验数涺进行必要的处理: 去掉均值, 消除传感器输出信号中的直流分量; 设置低通 滤波器, 解决高频千就问题.

本文编制的程序具有自动辨识模型阶次和参数的功能,同时又可以人为设定阶次、由低向 高地进行建模. 估计出差分方程系数后, 自动求解, 并绘图, 与实验曲线比较, 检验效果. 针对 第二组实验的同向输人、输出信号, 分别辨识出二阶和八阶差分方程形式的数学模型, 见图 1 、 2 所示. 可见, 用二阶系统等效腕力传感器是不合实际的. 随着模型阶次的提高, 理论值与实 测值之间的误差亚来愈小. 当模型阶数提高到八阶时, 辨识结果与实际曲线已相当吻合. 这 说明腕力传感器是个高阶系统.

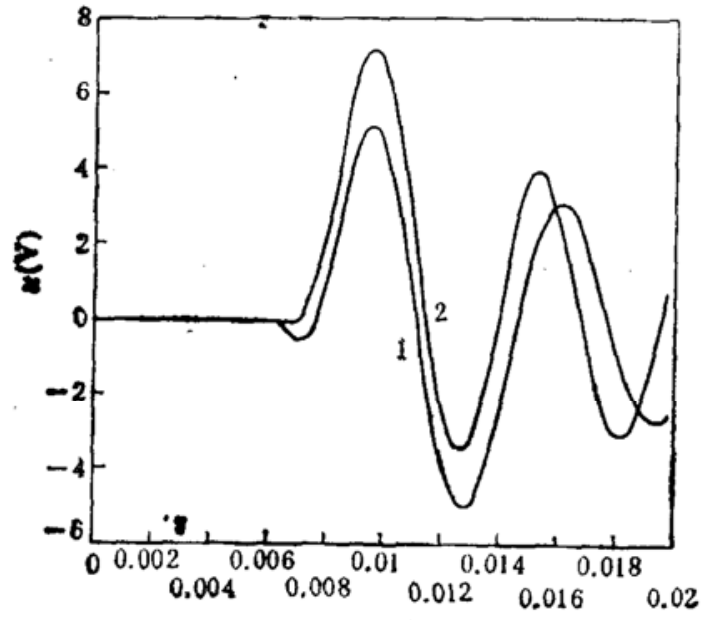

$t(\mathrm{~s})$

图 1

1 为理论值, 2 为实验值

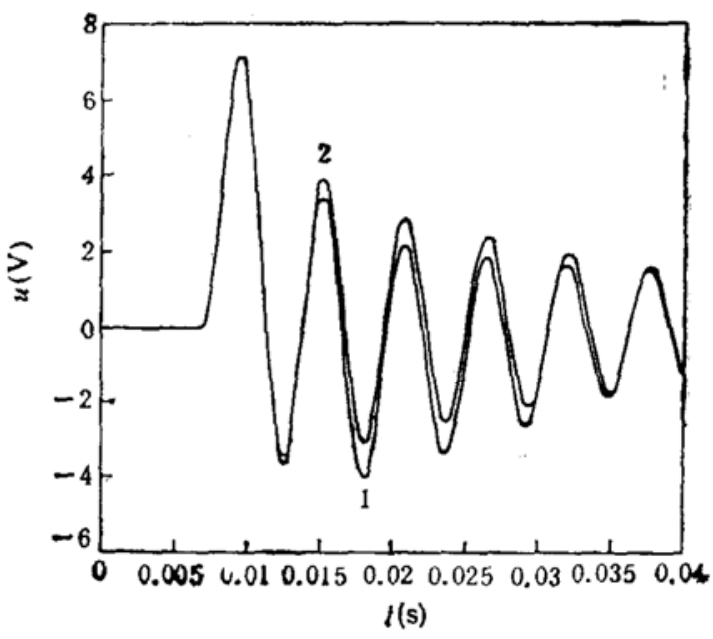

图 2

1,2 同图 1

\section{3 时序分析法建模}

在动态标定中, 传感器的输出信号是一连串随时间变化的有序数据, 即为时间序列. 时序 分析是用概率统计方法分析动态数据, 其中包括建模、参数估计和预测等 ${ }^{[2]}$. 它的特点是先找 合出数学模型,再利用此模型对系统进行研究. 时序分析中常用模型有三种, 本文建立㗮力传 感器的自回归 (AR) 全极点模型. 这是因为:

1. 传感器的输出数据是在动态标定时获取的, 基本上是确定性信号;

2. 在实验室条件下得到的动态数据,信噪比较高;

3. 用 AR 模型拟合动态数据的过程是线性的, 可用高效率的算法实现. 即它的参数估计 最为简单、预测计算速度最快。 
在 AR 模型中, 动态数据的现时值 $x_{m}$ 是用一步预测法从它的过去值 $x_{m-1}, x_{m-2}, \cdots, x_{m-N}$ 进行有限的组合得到, 即

$$
\hat{x}_{m}=a_{1} x_{m-1}+a_{2} x_{m-2}+\cdots+a_{N} x_{m-N}=\sum_{k=1}^{N} a_{k} x_{m-k} .
$$

实际上,真值 $x_{m}$ 等于估值 $\hat{x}_{m}$ 加上一微小的预测误差 $e_{m}$, 即

$$
x_{m}=\hat{x}_{m}+e_{m},
$$

式中 $e_{m}$ 为零均值的白噪声.

AR 模型拟合动态数据存在最佳阶数. 当模型阶次低于最佳数时, 会产生平滑效果; 而当 阶次高于最佳数时, 会导致结果的急剧变化和振荡. 采用最终预报误差准则 (FPE) 进行模 型阶数的判别, 即

$$
\mathrm{FPE}=\frac{N+m-1}{N-m-1} P_{m},
$$

式中 $N$ 为信号采样点数, $m$ 为 $\mathrm{AR}$ 模型阶数, $P_{m}$ 为预报误差功率. 采用伯格 (Burg) 递推算 法估计 AR 模型的参数. 该算法由低一阶模型参数递推计算高一阶模型的参数.

从第一组实验数据中,截取与输入同向的 100 个输出数据进行建模. 当定最高阶数 20 时, 运算表明 11 阶最佳; 将其理论值与实验值相比较, 不够吻合. 再定最高阶数为 30 时, 则 30 阶最佳, 其理论与实验曲线如图 3 所示. 截取第二组实验数据中的 100 个输出数据, 当定 最高阶数为 40 时, 16 阶最佳, 其理论与实验曲线如图 4 所示. 上两图中, 曲线 1 为理论值, 曲 线 2 是实测值. 可见, 用时序法建立传感器 AR 模型十分有效.

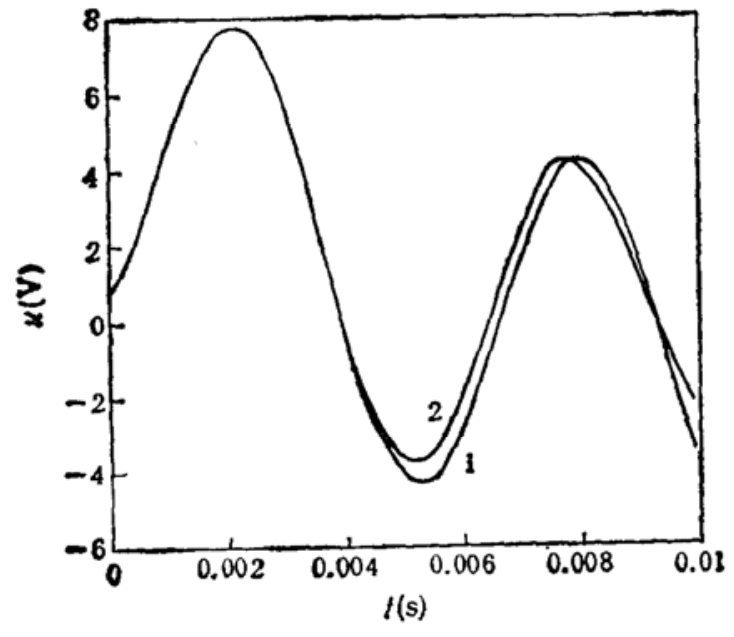

图 3

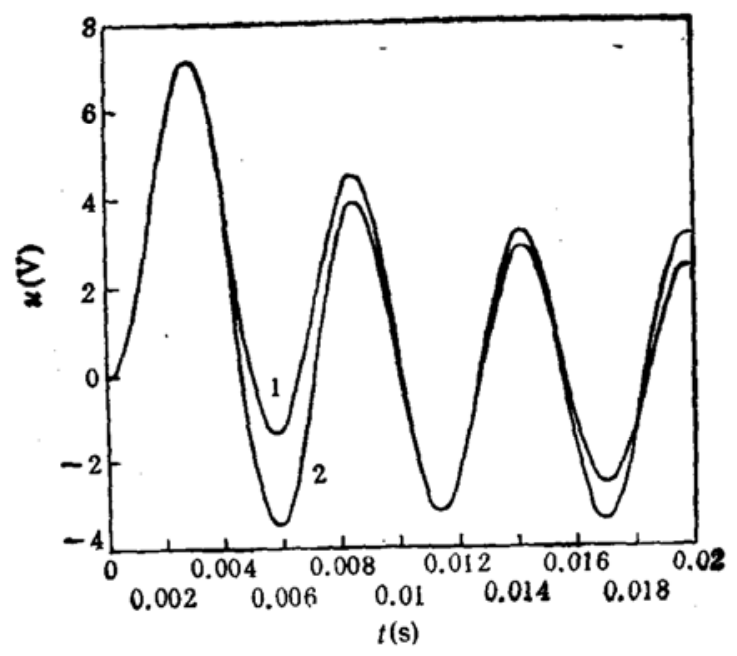

图 4 1,2 同图 1

\section{4 结 束 语}

本文从时域角度, 分别采用系统辩识和时序分析方法建立多维腕力传感器的动态数学模 型, 与实测相吻合. 这表明, 腕力传感器的动态模型是个高阶系统, 以往用二阶系统来等效是 不准确的, 由此定义的性能指标也不合理. 我们依据所建立的动态模型, 进行了数字仿真和动 态补偿, 大幅度改善了传感器的快速性. 
系统辨识法依据传感器输人、输出信号, 建模精度高, 例如, 以最佳阶数建模际, 辨识法的 最大相对误差是 $6.6 \%$, 时序法为 $8.1 \%$. 它得到差分方程形式的数学模型, 易于动态补偿, 但 存在 “数据饱和”问题. 应选择合适的采样频率; 或用多点抽一的方法选取数据. 时序分析法 建模只需传感器的输出数据, 当传感器不便进行动态标定或标定时输人数据不可测时, 此方法 有效. 在建模同时, 它可进行现代谱分析.

致谢作者感谢中国科学院合肥智能机械研究所的热情帮助.

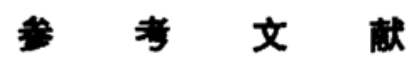

[1] Claudio Narduzzi, Carlo Offelli, IEEE Trans. Instrum. Meas., 1991, 40(2): 415-419.

[2] Lübke, P. J., Pinnow, K. M., Meas. Sci. Technol., 1991, 2:497--503. 